\title{
Search for Fractional Charges in Niobium and Tungsten
}

\author{
R. G. Milner, B. H. Cooper, ${ }^{(a)}$ K. H. Chang, K. Wilson, J. Labrenz, and R. D. McKeown \\ W. K. Kellogg Radiation Laboratory, California Institute of Technology, \\ Pasadena, California 91125 \\ (Received 10 January 1985)
}

\begin{abstract}
We have searched in niobium and tungsten for fractionally charged particles with effective nuclear charge $Z=N+\frac{1}{3}$ (where $N=0,1,2,3, \ldots$ ). In addition, we have looked for fractionally charged particles with $Z=\frac{2}{3}$ and $\frac{5}{3}$ in the same materials. No positive signal was observed, and concentration limits are reported.
\end{abstract}

PACS numbers: $14.80 . \mathrm{Dq}, 41.90 .+\mathrm{e}$

The reported observation ${ }^{1}$ of fractional charge on superconducting niobium spheres has stimulated much experimental ${ }^{2,3}$ and theoretical ${ }^{4,5}$ work in the past few years. Aside from Ref. 1, the experiments that search in niobium ${ }^{2}$ all assumed that the fractionally charged particles (FCP) will diffuse out of the sample as a positive ion when the sample is heated. The efficiency of this diffusion process is difficult to estimate. The effects observed by the authors of Ref. 1 could imply the existence of fractional charges (modulo $\frac{1}{3} e$ ) at a concentration level of $2 \times 10^{-18} / \mathrm{Nb}$ atom, and possibly that the fractional charge is transferred from tungsten during annealing of the niobium sample. Since most of the residual charges observed corresponded to $+\frac{1}{3} e$ (rather than $-\frac{1}{3} e$ ) a fractionally charged particle with an effective nuclear charge of $Z=N+\frac{1}{3}$ (where $N=0,1,2,3, \ldots)$ is more likely than those with $Z=N+\frac{2}{3}$. We define the quantity $Z$ as the charge that would remain on the FCP if all of the atomic electrons were removed.

We have performed an experimental search to test this hypothesis, and report the initial results in this Letter. Our technique is sensitive to FCP with $Z=N+\frac{1}{3} \quad(N=0,1,2,3, \ldots)$ which would form negative ions of charge $-\frac{2}{3}$, as well as FCP with $Z=\frac{2}{3}$ or $\frac{5}{3}$ which would form negative ions of charge $-\frac{1}{3}$. A detailed paper describing the apparatus and technique will be published elsewhere.

The method we employed involves four steps (see Fig. 1): (a) extraction of the FCP from the host sample and formation of a negative ion (see below); (b) acceleration of negative FCP and stripping of an electron in a tandem electrostatic accelerator; (c) deflection of the (now positively charged) FCP in a transverse electric field; and (d) measurement of the kinetic energy in a particular detector. The acceleration and deflection of nonrelativistic charged particles from rest in purely electrostatic fields yields trajectories which are mass independent (stray magnetic fields and relativistic effects limit our sensitivity to FCP with mass $\geq 200 \mathrm{MeV} / c^{2}$ ). The efficiency for the transmission of particles through the accelerator to a particular final charge state is primarily determined by the stripping efficiency, which drops off for masses greater than several hundred $\mathrm{GeV} / c^{2}$. The transmission efficiencies and mass independence of trajectories were determined by use of integrally charged ion beams $\left(1 \mathrm{GeV} / c^{2}<m<200 \mathrm{GeV} / c^{2}\right)$ from both the FCP source and a conventional sputter ion source.

The charge measurement results from steps (c) and (d). Step (c) selects ions with the desired ratio of kinetic energy to charge (the "electric rigidity") to an

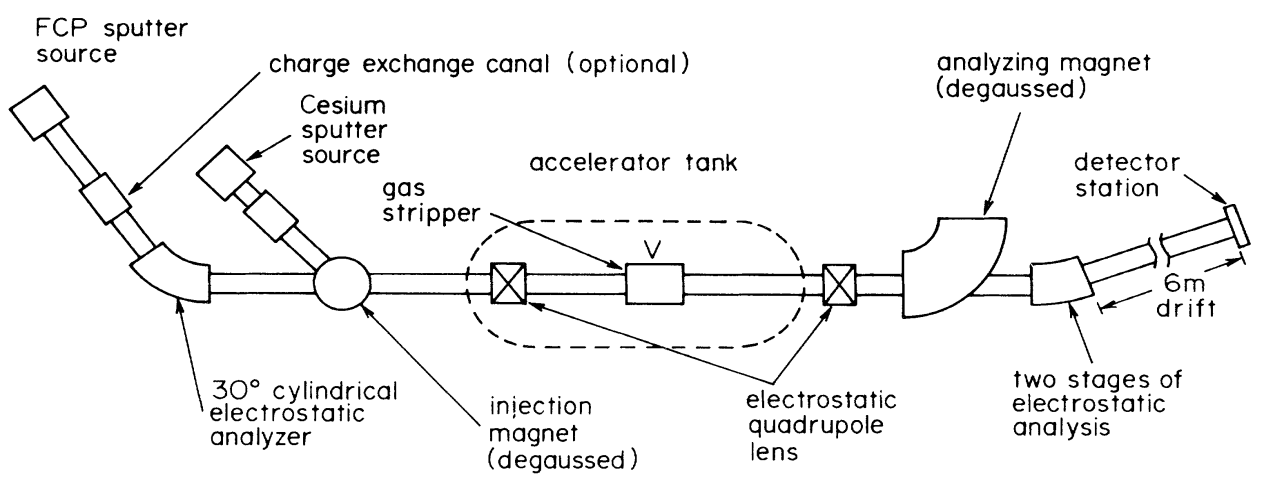

FIG. 1. Schematic diagram of the experimental apparatus. 
accuracy of $0.2 \%$ and step (d) measures the kinetic energy, so that the charge is uniquely determined for each detected particle. Background counts arise from rare scattering and charge-exchange processes in the acceleration and transport system. The transmission efficiency of the accelerator and beam transport system is $3 \%-50 \%$, depending upon the mass and nuclear charge of the FCP.

Particles were extracted from the host sample by sputtering with a magnetically analyzed $30-\mathrm{keV} \mathrm{Ar}^{+}$ beam in a vacuum chamber at a pressure of $\sim 10^{-8}$ Torr. The sample was sputtered at a rate of $\sim 10^{15}$ atoms/sec (from a 1-mm-diam area) and the negative-ion yield was typically $2-3 \mathrm{nA}$. We have verified that essentially $100 \%$ of the sputtered ions are collected and then preaccelerated by $70 \mathrm{kV}$ of potential.

For fractional charges such as $Z=\frac{1}{3}$ with low electron affinity (i.e., less than the work function of the host material), the probability of negative-ion formation in the sputtering process is very small. Therefore, in a separate experiment, we extracted the positive ions and passed them through a rubidium vapor to attach an electron before acceleration. The probability of electron pickup was estimated to be $20 \%-60 \%$ (depending on the mass $Z$ of the FCP) by use of electron affinities given by Lackner and Zweig ${ }^{4}$ in semiempirical formulas. ${ }^{6}$

A well-defined "beam" of particles was found to arise from ions that emerge from the accelerator with charge +2 and then pick up an electron from a residual gas atom (to form +1 ions) before reaching the electrostatic analyzer. This "beam" of $\sim 10^{6}$ particles/sec differs in electric rigidity by only $1.1 \%$ from FCP which strip from $-\frac{2}{3}$ to $+\frac{1}{3}$ in the gas stripper of the tandem accelerator, and was used as a benchmark to aid in the setting up of the electrostatic analyzer for those runs.

The search for penetrating FCP $(Z<2)$ was conducted with a $\Delta E-E$ detector telescope consisting of a gas ionization chamber followed by a silicon surfacebarrier detector. A second silicon counter served to identify any very penetrating particles. For heavyion-type FCP $(Z>2)$ we employed a $20-\mu$ m-thick heavy-ion silicon detector, allowing for "pulse-height defect" ${ }^{\prime \prime}$ in the data analysis. Lead shielding and active plastic-scintillator veto counters were often necessary to suppress room background and/or cosmicray-associated events.

The FCP which strip from $-\frac{1}{3}$ to $+\frac{2}{3}$ in the gas stripper are degenerate in electric rigidity with ions which strip from -1 to +2 . The +2 beam is a heavy-ion beam (i.e., $Z>2$ ). We searched for penetrating $(Z<2)$ FCP by using a $3.4-\mathrm{mg} / \mathrm{cm}^{2}$ aluminium absorber foil in front of the detector to eliminate the high flux of these heavy ions. This resulted in a manageable count rate in the detector. The remaining observed particles are in fact protons from $\mathrm{H}_{2}^{-}$ions which are dissociated in the gas stripper.

No positive evidence was found for the presence of fractional charges in either niobium or tungsten. In Table I we summarize our results for niobium. The limits reported vary for the different species because the accelerator transmission, background rate, and running time depended on the experimental configuration (extraction polarity, stripper gas, and detector) and type of FCP assumed in the search.

For FCP with $Z>2$, concentration limits are calculated by two different methods: (1) Total background rate in the pulse-height window is converted to a concentration ("Integral"), and (2) the level at which a statistically significant peak $(1 \sigma)$ would be evident in the pulse-height spectrum ("Peak") is also computed. Method (2) is valid under the assumption that the FCP are all of one species, i.e., same mass and nuclear charge. Method (1) applies if one assumes that a variety of FCP species are present.

For $Z=\frac{1}{3}$, the concentration limit has a significant mass dependence due to the velocity dependence of the electron pickup probability in the rubidium vapor and the energy dependence of the background rate in the detector system. Therefore, this limit is displayed in Fig. 2(a) as a function of mass. These effects were

TABLE I. Niobium concentration limits.

\begin{tabular}{|c|c|c|c|}
\hline \multirow[b]{2}{*}{$Z$} & \multirow{2}{*}{$\begin{array}{c}\text { Mass } \\
\left(\mathrm{GeV} / \mathrm{c}^{2}\right)\end{array}$} & \multicolumn{2}{|c|}{$\mathrm{FCP} /$ niobium atom } \\
\hline & & Peak & Integral \\
\hline$\frac{1}{3}$ & $0.2 \leq m \leq 250$ & See Fig. 2(a) & \\
\hline$\frac{2}{3}$ & $0.2 \leq m \leq 250$ & $2.2 \times 10^{-17}$ & \\
\hline$\frac{4}{3}$ & $0.3 \leq m \leq 14$ & $5.2 \times 10^{-19}$ & \\
\hline$\frac{5}{3}$ & $0.2 \leq m \leq 4$ & $4.9 \times 10^{-18}$ & \\
\hline$N+\frac{1}{3} a$ & $0.7 \leq m \leq 250$ & $1.5 \times 10^{-18}$ & $2.8 \times 10^{-17}$ \\
\hline$N+\frac{1}{3} \mathrm{~b}$ & $0.7 \leq m \leq 250$ & See Fig. 2(b) & $1 \times 10^{-17}$ \\
\hline
\end{tabular}

${ }^{\mathrm{a}} N=1,2,3, \ldots$ and electron affinity $\geq 4 \mathrm{eV}$; negative extraction.

${ }^{\mathrm{b}} N=1,2,3, \ldots$ and electron affinity $\leqslant 4 \mathrm{eV}$; positive extraction with rubidium charge exchange. 
TABLE II. Tungsten concentration limits.

\begin{tabular}{clcr}
\hline \hline & \multicolumn{1}{c}{ Mass } & \multicolumn{2}{c}{ FCP/tungsten atom } \\
$\left(\mathrm{GeV} / c^{2}\right)$ & Peak & Integral \\
\hline$\frac{1}{3}$ & $0.2 \leq m \leq 250$ & See Fig. 2(a) & \\
$\frac{2}{3}$ & $0.2 \leq m \leq 250$ & $1.2 \times 10^{-16}$ & \\
$\frac{4}{3}$ & $0.3 \leq m \leq 14$ & $5.9 \times 10^{-18}$ & $4 \times 10^{-16}$ \\
$\frac{5}{3}$ & $0.2 \leq m \leq 4$ & $1.3 \times 10^{-17}$ & $2.5 \times 10^{-17}$ \\
$N+\frac{1}{3}$ a & $0.7 \leq m \leq 250$ & $1 \times 10^{-16}$ & \\
$N+\frac{1}{3}$ b & $0.7 \leq m \leq 250$ & See Fig. 2(b) \\
\hline \hline
\end{tabular}

${ }^{\mathrm{a}} N=1,2,3, \ldots$ and electron affinity $Z 4 \mathrm{eV}$; negative extraction.

${ }^{\mathrm{b}} N=1,2,3, \ldots$ and electron affinity $\leq 4 \mathrm{eV}$; positive extraction with rubidium charge exchange.

also important for $Z=N+\frac{1}{3}(N=1,2,3, \ldots)$ when positive extraction was employed in the ion source (i.e., for FCP with low electron affinity). Therefore, these results are similarly graphed in Fig. 2(b).

The quoted concentration limits are actually estimates since the transmission efficiencies and sputter rates of FCP are inferred from the behavior of integrally charged ions. In general these estimates are probably accurate to about a factor of 2 ; somewhat

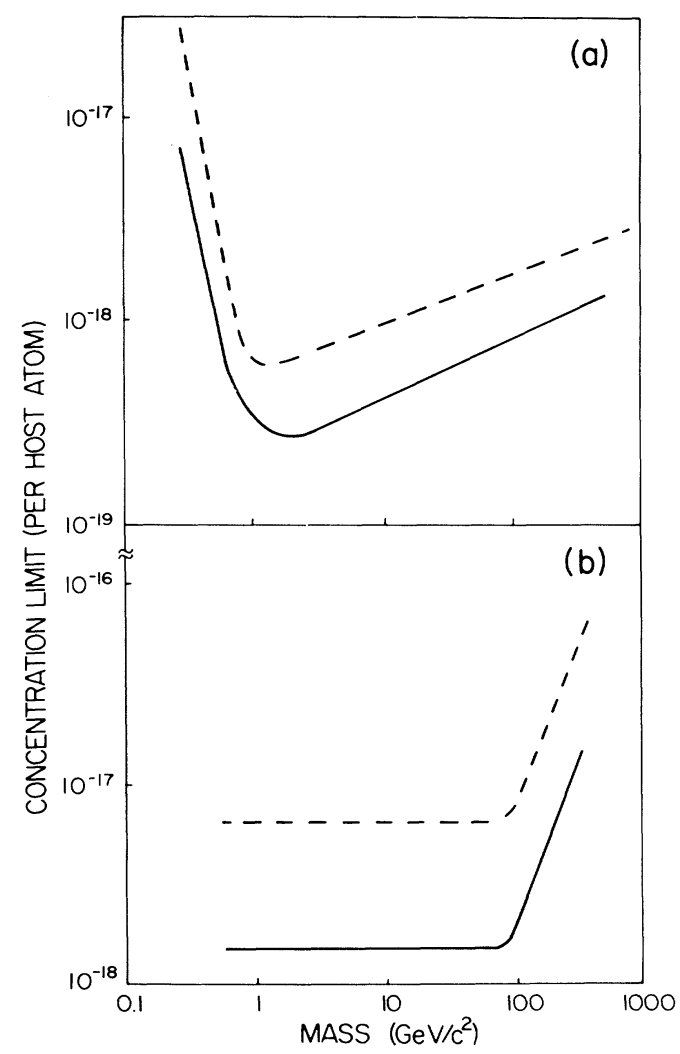

FIG. 2. Upper limit for concentration of FCP with (a) $Z=\frac{1}{3}$ and (b) $Z>2$ with low electron affinity. The solid line is the limit for niobium, and the dashed line is for tungsten. larger deviations are conceivable in special cases. Analogous results for the FCP search in tungsten are given in Table II and Fig. 2.

The present results severely constrain the plausible interpretations of the Stanford experiment. ${ }^{1}$ In particular, it appears that a heavy-ion-type FCP with $Z=N+\frac{2}{3}(N=2,3,4, \ldots)$ is the only remaining candidate that could be present in appreciable quantities in niobium (i.e., greater than a few FCP per ball). The explanation proposed by Morgan and Barnhill ${ }^{8}$ requires large concentrations $\left(\sim 10^{-14}\right.$ per $\mathrm{W}$ atom) of FCP in tungsten compared to the upper limits reported in this Letter.

By means of our technique, it would be quite difficult to improve substantially the limits obtained here. Therefore, it appears most fruitful to expand our search to some other promising materials, such as meteorite samples. We also plan to examine some samples exposed to reaction products from relativistic heavy-ion collisions and possibly teraelectronvolt proton collisions with heavy nuclei.

This research was supported by the National Science Foundation under Grant No. PHY82-15500 and by the California Institute of Technology Sloan Fund.

(a) Present address: Physics Department, Cornell University, Ithaca, N.Y. 14853.

${ }^{1}$ G. S. LaRue, J. D. Phillips, and W. M. Fairbank, Phys. Rev. Lett. 46, 967 (1981).

2J. P. Schiffer et al., Phys. Rev. D 17, 2241 (1978); W. Kutschera et al., Phys. Rev. D 29, 791 (1984).

${ }^{3}$ C. L. Hodges et al., Phys. Rev. Lett. 47, 1651 (1981); D. C. Joyce et al., Phys. Rev. Lett. 51, 731 (1983); M. A. Lindgren et al., Phys. Rev. Lett. 51, 1621 (1983); W. Marinelli and G. Morpurgo, Phys. Rep. 85, 161 (1982).

${ }^{4}$ K. S. Lackner and G. Zweig, Phys. Rev. D 28, 28 (1983).

${ }^{5 R}$. Slansky, T. Goldman, and G. L. Shaw, Phys. Rev. Lett. 47, 887 (1981).

6J. B. Hasted, Adv. Electron. Electron Phys. 13, 1 (1960).

${ }^{7}$ S. B. Kaufman et al., Nucl. Instrum. Methods 115, 47 (1974).

8J. D. Morgan, III, and M. V. Barnhill, III, Phys. Lett. 133B, 227 (1983). 